\title{
CORRELATION BETWEEN VISUAL GAIT ANALYSIS AND FUNCTIONAL ASPECTS IN CEREBRAL PALSY
}

\author{
Maira Rech Folle ${ }^{1}$, Ana Paula Tedesco ${ }^{1}$, Renata $D^{\prime}$ Agostini Nicolini-Panisson ${ }^{1}$ \\ 1. Instituto de Neuro-Ortopedia, Caxias do Sul, RS, Brazil.
}

\section{ABSTRACT}

Objective: To verify the correlation between visual gait analysis (VGA) by the Edinburgh visual gait score (EVGS) and functional aspects using the Timed Up and Go Test (TUG) and Gross Motor Function Classification System (GMFCS) in individuals with cerebral palsy (CP). Methods: Retrospective cross sectional study of 35 patients with CP. The mean age 12.61 years old, 94.3\% were spastic; $34.4 \%$ hemiplegic, $54.3 \%$ diplegic, $11.4 \%$ triplegic; $45.7 \%$ were level II GMFCS, $42.9 \%$ level I, 5.7\% level III and 5.7\% level IV. VGA was analyzed by the Edinburgh visual gait score (EVGS), functional mobility was assessed by TUG and functionality through GMFCS. The Spearman correlation was used for statistical analysis. Results: The mean EVGS score was 18.97. The mean TUG was $13.71 \mathrm{sec}$. EVGS showed moderate correlation with TUG $(r=0.46, p=0.03)$ and GMFCS ( $r=0.45$, $\mathrm{p}=0.00$ ). Conclusion: Worse VGA scores correlate to worse functionality and mobility performance. Due to the observed correlation, it is possible to assert that VGA is a useful tool on evaluation of CP patients. Level of Evidence III, Retrospective Comparative Study.

Keywords: Gait. Cerebral palsy. Evaluation.

Citation: Folle MR, Tedesco AP, Nicolini-Panisson RD. Correlation between visual gait analysis and functional aspects in cerebral palsy. Acta Ortop Bras. [online]. 2016;24(5):259-61. Available from URL: http://www.scielo.br/aob.

\section{INTRODUCTION}

The gait analysis is an important tool for assessing changes in gait of neurological patients. The gold standard for evaluation is computadorized gait analysis (CGA).

The importance of gait assessment in cerebral palsy (CP) has been found in a study that showed that CGA has a strong impact on surgical treatment decision and that worst results were recorded by surgeons who did not follow what was proposed by CGA. ${ }^{1}$ CGA, however, is an expensive and unavailable technology in many centers, so that visual gait analysis (VGA) with video recording may assist the process of study, diagnosis and treatment indication. ${ }^{2}$ VGA is indicated for normal and pathological gait assessment, whenever CGA is not available; in very young children, under 4 to 6 years old; and in individuals with little comprehension and cooperation. ${ }^{1-3}$

One of the disadvantages that have been described regarding VGA is the difficulty in visualizing the transverse plane. However, the placement of rotation indicators (markers) in the pelvis and thigh may aid visualization of pelvis and hip rotations. ${ }^{4}$

The literature shows that even CGA, which provides accurate and objective parameters, relies on subjective data interpretation and is, therefore, variable, especially if they are analyzed in different laboratories, also depending on the examiners' experience and reliability of clinical examination and data collection techniques. ${ }^{5-7}$
CGA can still show differences in the results due to errors of the own computer system and software, markers placement technique, or slight performance variability of individuals between tests. ${ }^{8}$ Thus, even if VGA is unable to discriminate the quality of movement as CGA, it is considered a useful tool of moderate reliability, which is greatly influenced by the clinical experience of the observer. ${ }^{2,9-11}$ There are several protocols described in the literature to evaluate the VGA in CP, such as the Physician's Rating Scale (PRS), ${ }^{12}$ the Edinburgh Visual Analysis Score (EVGS) ${ }^{13}$ and the Observational Gait Scale, ${ }^{14}$ among others.

EVGS was developed as a VGA score for patients with CP. Gait videos in coronal and sagittal planes are analyzed according to 17 parameters for each inferior limb, which correspond to key elements of normal and pathological gait, graduates in a three scores range (0: normal, 1 : moderate and 2 : marked), the maximum score being 34. Six different anatomical levels are analyzed: trunk, pelvis, hip, knee, ankle and foot, in the transverse, coronal and sagittal planes and support and balance phases of the gait. ${ }^{13}$ EVGS had its inter and intraobserver reliability and sensitivities evaluated in studies that compared it with CGA, with a correlation around $64 \%$ of the items assessed. ${ }^{13}$ It has also been compared to other measurements, such as the Gillette Gait Index, Gillette Functional Assessment Questionnaire and speed, and showed significant correlations, especially with Gillette Gait Index. ${ }^{15}$

All the authors declare that there is no potential conflict of interest referring to this article. 
The intra and inter-observer reliability of the scale depends on the observers' experience and training - the higher the experience, the greater the intraobserver reliability. ${ }^{16-20} \mathrm{~A}$ study concluded that the observation of gait events by inexperienced observers using EVGS was moderately reliable, however, there was little precision when compared to experienced observers and to CGA. ${ }^{19}$ Another study comparing PRS and EVGS, showed excellent intraobserver reliability, but the inter-observer reliability for both scales was considered low. The study recommended that VGA was made by the same observer. ${ }^{16}$

The reliability and validity of EVGS and five other tools was compared with the CGA. EVGS was considered the best tool to assess the gait pattern in $\mathrm{CP}$, because it considers motion data in the three planes, with good reliability and concurrent validity. 4,19-22 There are studies that correlate data from VGA with functional mobility measurements and levels of functionality. There are a correlation between data from CGA and EVGS and the Gross Motor Function Classification System levels (GMFCS), demonstrated that high scores on both gait assessment methods matched with high levels of GMFCS. ${ }^{21}$ In another study, the Timed Up \& Go method (TUG) was analyzed, along with other functional mobility elements and showed strong correlation with functional gait ability of individuals with $\mathrm{CP} .{ }^{23} \mathrm{Kerr}$ et al. ${ }^{24}$ have shown that low levels of GMFCS were associated to low values of the Pediatric Evaluation of Disability Inventory.

GMFCS was developed to classify the functional abilities of children with CP. This scale is currently considered the most reliable and best known in pediatric CP rehabilitation. ${ }^{25}$

TUG is a quick and easy test that assesses the functional mobility and consists in measuring the time spent to go from the sitting position to orthostatic posture, start walking at the command, stop, return, come back, and sit down again. This test was validated and adapted for children and adolescents with $\mathrm{CP}^{26}$ and has also has normal values for children and adolescents. ${ }^{27}$ With this perspective, the objective of this study was to verify the correlation between gait pattern measured by EVGS, functional mobility (TUG) and the level of functionality (GMFCS) of individuals with CP.

\section{MATERIALS AND METHODS}

This is a cross-sectional retrospective review study of medical records of patients with CP who underwent VGA analyzed using EVGS between January 2010 and March 2015, as part of their evaluation at Instituto de Neuro-Ortopedia. The records with incomplete data were excluded. This study was approved by the Research Ethics Committee under number 1127171 (CAAE: 43305015.5.0000.5668). In the VGA assessment routine, anatomical marks were made to facilitate observation of the videos. Besides marks made with white paint on the patella and posterior side of the calcaneus, as well as markers in the anterior-superior iliac crests, as described in the original study, ${ }^{13}$ markers in the sacrum and dorsal surface of the feet (at the level of the second metatarsal), respectively to better identify deviations in the transverse and coronal plane of the legs ${ }^{4}$ For individuals showing difficulty to walk in a straight line, markers were placed on the floor with tape for the purpose of orientation. In this study, the data collected from VGA, performance in the TUG test and classification by GMFCS were analyzed, aiming to demonstrate correlation between them. VGA was performed using videos in the coronal and sagittal planes with extra focus on the feet. The videos were analyzed using the criteria described in the EVGS and the total score was calculated by summing the score of both limbs.

The TUG test was conducted according to the methodology described by Williams. ${ }^{26}$ Three repetitions of the path were performed and the only the best time was considered. That is, the lower the number, the better the functional mobility.

GMFCS has five classification levels based on functional abilities and the CP child's movement initiative, emphasizing sitting and walking set by age groups. Distinctions between the levels are based on functional limitations, need for assistive technology, including the use of assistive devices (crutches, walkers and canes) and wheelchair. ${ }^{25}$

\section{Statistical analysis}

For statistical purposes, the normality of continuous variables was assessed by the Shapiro-Wilk test. Data with normal distribution were presented as mean and standard deviation and asymmetric data with median and interquartile range. Categorical variables were expressed as absolute and relative frequency. The association between the total Edinburgh score (sum of both lower limbs) with GMFCS and TUG was performed using Spearman's correlation test. All analysis and data processing were performed using SPSS version 18.0 (SPSS Inc., USA). In all cases, differences were considered significant when $p<0.05$.

\section{RESULTS}

The review included data from 35 patients who underwent VGA and 28 subjects that were also evaluated through the TUG test. Table 1 shows the characteristics of the participants of the study. Both correlations of EVGS with TUG and GMFCS showed a moderate magnitude, according to Table 2.

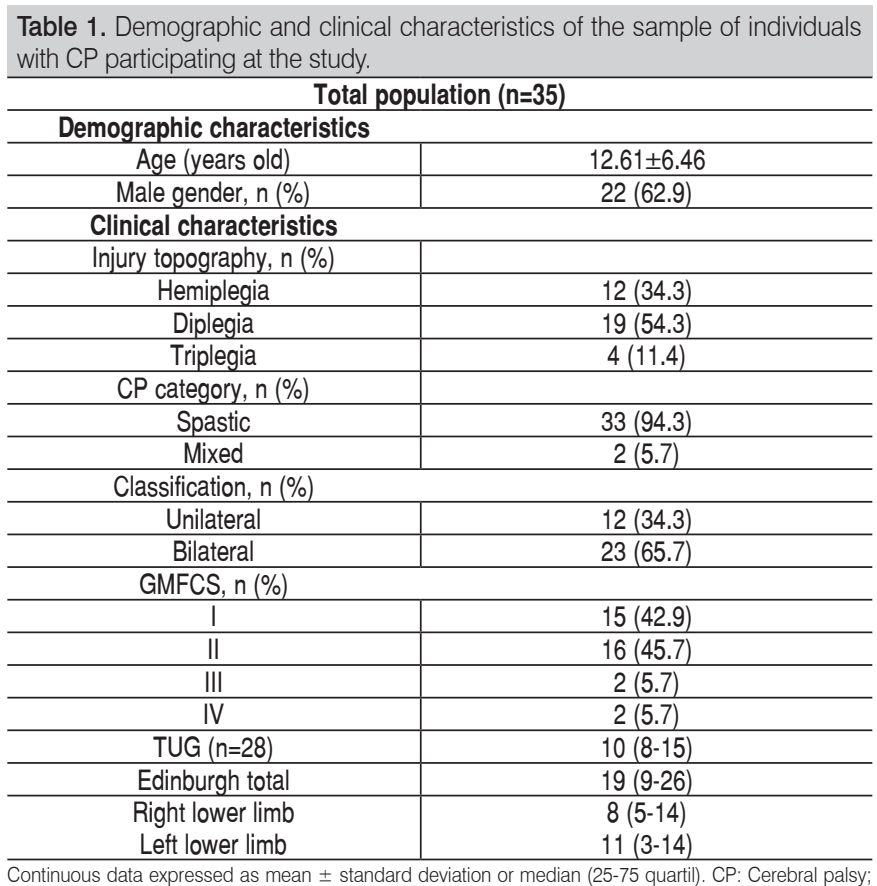
GMFCS: Gross Motor Function Classification System; TUG: Timed Up and Go test.

\begin{tabular}{|c|c|c|c|c|}
\hline & GMFCS & Magnitude & TUG & Magnitude \\
\hline Edinburgh & $0.45(0.00)$ & Moderate & $0.46(0.03)$ & Moderate \\
\hline
\end{tabular}
System; TUG: Timed Up and Go test. 


\section{DISCUSSÃO}

Correlations of EVGS with TUG and GMFCS, found in our study, were considered as moderate, showing the relationship between gait pattern, functional mobility and level of functionality. It has been shown that individuals with high scores on EVGS, i.e., major changes in gait pattern took more time to carry out the functional mobility test (TUG) and had worst level of functionality (GMFCS). Similar results were obtained by Robinson et al., ${ }^{21}$ who demonstrated a strong relationship between the results of CGA and EVGS, reinforcing the latter as an appropriate tool for examiners who do not have access to CGA. In this study, authors found a strong relationship between both CGA and EVGS with level of GMFCS I-III, and it has been observed that high gait scores were associated to high levels of GMFCS. ${ }^{21}$

A study that analyzed the gait performance through speed and the Gross Motor Function Measure (GMFM) demonstrated its relationship with the dimensions $D$ (standing) and $E$ (walking, running and jumping). The authors emphasized that the video recording enables better analysis of movements and assists the selection and evaluation of gait training strategies. ${ }^{28}$

The correlation between functional tests as TUG and the levels of GMFCS was demonstrated in a study that investigated the gait behavior in adult patients with CP. The authors reported decline in gait function, as compared with adolescents, and in 39\% of cases there was change in the level of GMFCS. A correlation between TUG, GMFCS and Functional Mobility Scale with the six-minute walk test, evaluated in the study, showing that TUG had a direct influence on the functional gait ability. ${ }^{23}$

The literature reports GMFCS findings correlate with other mobility scales, such as the Pediatric Evaluation of Disability Inventory, for example, demonstrating that the lower the GMFCS level, the lower the mobility score at the Pediatric Evaluation of Disability Inventory. ${ }^{24}$ However, this study did not directly analyzed gait abnormalities, as in our study.

\section{CONCLUSION}

It was possible to demonstrate that VGA, analyzed by the Edinburgh protocol, is able to correlate gait abnormalities with functional capacity, measured by the TUG test and GMFCS. Worse scores correlate with worse performance in terms of functionality and mobility in CP. EVGS seems to be an appropriate tool to evaluate the progress of patients with $\mathrm{CP}$.

AUTHORS' CONTRIBUTIONS: Each author contributed individually and significantly to the development of the study. All authors contributed to the writing of the manuscript. MRF (0000-0002-3990-2090)* reviewed the records for data collection. APT (0000-0001-5406-661X)* developed the study design, oriented data collection and analysis, oriented the statistical analysis, results analysis and collaborated for the development of the manuscript. RDNP (0000-0001-9728-3649)* evaluated the data of the statistical analysis. All authors contributed to the intellectual concept of the study. ${ }^{\star}$ ORCID (Open Researcher and Contributor ID).

\section{REFERENCES}

1. Ferrari A, Brunner R, Faccioli S, Reverberi S, Benedetti MG. Gait analysis contribution to problems identification and surgical planning in CP patients: an agreement study. Eur J Phys Rehabil Med. 2015;51(1):39-48.

2. Harvey A, Gorter JW. Video gait analysis for ambulatory children with cerebral palsy: Why, when, where and how! Gait Posture. 2011; 33(3):501-3.

3. Coutts F. Gait analysis in the therapeutic environment. Man Ther. 1999:4(1):2-10.

4. Hillman SJ, Hazlewood ME, Loudon IR, Robb JE. Can transverse plane rotations be estimated from video tape gait analysis? Gait Posture. 1998;8(2):87-90.

5. 5.Skaggs DL, Rethlefsen SA, Kay RM, Dennis SW, Reynolds RA, Tolo VT. Variability in gait analysis interpretation. J Pediatr Orthop. 2000;20(6):759-64.

6. Noonan KJ, Halliday S, Browne R, O'Brien S, Kayes K, Feinberg J. Interobserver variability of gait analysis in patients with cerebral palsy. J Pediatr Orthop. 2003;23(3): 279-87

7. 7.Cook RE, Schneider I, Hazlewood ME, Hillman SJ, Robb JE. Gait analysis alters decision-making in cerebral palsy. J Pediatr Orthop. 2003;23(3):292-5.

8. 8.Chambers C, Goode B. Variability in gaitmeasurementsacrossmultiple sites. Gait Posture. 1996;4:167-208.

9. Krebs DE, Edelstein JE, Fishman S. Reliability of observational kinematic gait analysis. Phys Ther. 1985;65(7):1027-33.

10. Eastlack ME, Arvidson J, Snyder-Mackler L, Danoff JV, McGarvey CL. Interrater reliability of videotaped observational gait-analysis assessments. Phys Ther 1991:71(6):465-72.

11. Brunnekreef JJ, van Uden CJ, van Moorsel S, Kooloos JG. Reliability of videotaped observational gait analysis in patients with orthopedic impairments.BMC Musculoskelet Disord. 2005:6:17

12. Koman LA, Mooney JF 3rd, Smith BP, Goodman A, Mulvaney T. Management of spasticity in cerebral palsy with botulinum-A toxin: report of a preliminary, randomized, double-blind trial. J Pediatr Orthop. 1994;14(3):299-303.

13. Read HS, Hazlewood ME, Hillman SJ, Prescott RJ, Robb JE. Edinburgh visual gait score for use in cerebral palsy. J Pediatr Orthop. 2003;23(3):296-301.

14. Araujo PA, Kirkwood RN, Figueiredo EM. Validade e confiabilidade intra e inter-examinadores da Escala Observacional de Marcha para crianças com paralisia cerebral espástica. Rev Bras Fisioter. 2009;13(3):267-73.

15. Hillman SJ, Hazlewood ME, Schwartz MH, van der Linden ML, Robb JE. Correlation of the Edinburgh Gait Score with the Gillette Gait Index, the Gillette Functional Assessment Questionnaire, and dimensionless speed. J Pediatr Orthop. 2007;27(1):7-11.

16. Maathuis KG, van der Schans CP, van Iperen A, Rietman HS, Geertzen JH.
Gait in children with cerebral palsy: observer reliability of Physician Rating Scale and Edinburgh Visual Gait Analysis Interval Testing scale. J Pediatr Orthop. 2005;25(3):268-72.

17. Dickens WE, Smith MF. Validation of a visual gait assessment scale for children with hemiplegic cerebral palsy. Gait Posture. 2006;23(1):78-82

18. Brown CR, Hillman SJ, Richardson AM, Herman JL, Robb JE. Reliability and validity of the Visual Gait Assessment Scale for children with hemiplegic cerebral palsy when used by experienced and inexperienced observers. Gait Posture. 2008;27(4):648-52.

19. Ong AM, Hillman SJ, Robb JE. Reliability and validity of the Edinburgh Visual Gait Score for cerebral palsy when used by inexperienced observers. Gait Posture. 2008;28(2):323-6.

20. Viehweger E, Zürcher Pfund L, Hélix M, Rohon MA, Jacquemier M, Scavarda $D$, et al. Influence of clinical and gait analysis experience on reliability of observational gait analysis (Edinburgh Gait Score Reliability). Ann Phys Rehabil Med. 2010;53(9):535-46

21. Robinson LW, Clement N, Fullarton M, Richardson A, Herman J, Henderson $\mathrm{G}$, et al. The relationship between the Edinburgh Visual Gait Score, the Gait Profile Score and GMFCS levels I-III. Gait Posture. 2015;41(2):741-3.

22. Rathinam C, Bateman A, Peirson J, Skinner J. Observational gait assessment tools in paediatrics--a systematic review. Gait Posture. 2014;40(2):279-85.

23. Maanum G, Jahnsen R, Frøslie KF, Larsen KL, Keller A. Walking ability and predictors of performance on the 6-minute walk test in adults with spastic cerebral palsy. Dev Med Child Neurol. 2010;52(6):e126-32.

24. Kerr C, McDowell BC, Parkes J, Stevenson M, Cosgrove AP. Age-related changes in energy efficiency of gait, activity, and participation in children with cerebral palsy. Dev Med Child Neurol. 2011;53(1):61-7.

25. Palisano R, Rosenbaum P, Walter S, Russell D, Wood E, Galuppi B. Development and reliability of a system to classify gross motor function in children with cerebral palsy. Dev Med Child Neurol. 1997;39(4):214-23.

26. Williams EN, Carroll SG, Reddihough DS, Phillips BA, Galea MP. Investigation of the timed 'up \& go' test in children. Dev Med Child Neurol. 2005;47(8):518-24

27. Nicolini-Panisson RD, Donadio MV. Normative values for the Timed 'Up and Go'test in children and adolescents and validation for individuals with Down syndrome. Dev Med Child Neurol. 2014;56(5):490-7.

28. Drouin LM, Malouin F, Richards CL, Marcoux S. Correlation between the gross motor function measure scores and gait spatiotemporal measures in children with neurological impairments. Dev Med Child Neurol. 1996;38(11):1007-19. 